\title{
Option Pricing in an Exponential MixedTS Lévy process
}

\author{
Lorenzo Mercuri • Edit Rroji
}

Received: date / Accepted: date

\begin{abstract}
In this paper we present an option pricing model based on the assumption that the underlying asset price is an exponential Mixed Tempered Stable Lévy process. We also introduce a new $\mathrm{R}$ package called PricingMixedTS that allows the user to calibrate this model using procedures based on loss or likelihood functions.
\end{abstract}

Keywords Exponential Lévy process · Mixed Tempered Stable · R package . Calibration

\section{Introduction}

The main object of this paper is to present an option pricing model under the assumption that log returns are generated from a Lévy Mixed Tempered Stable (MixedTS hereafter) process. This process is built using the infinitely divisible property of the MixedTS distribution recently introduced by Rroji and Mercuri (2015) as a generalization of the Normal Variance Mean Mixture (NVMM hereafter) family of distributions.

The relevance of Lévy processes has been widely investigated in financial modeling especially for asset prices (Carr and Wu, 2004a; Eberlein and Madan, 2009; Carr and Madan, 1999, see for instance) mainly due to the fact that these processes are able to handle with the stylized facts. Moreover, they are preferred to stochastic volatility models as shown in Li et al. (2008) and in Tankov (2003). The reason for enlarging the family of Lévy processes by introducing the MixedTS arises from its ability to overcome some limits of the processes built on the NVMM extensively used

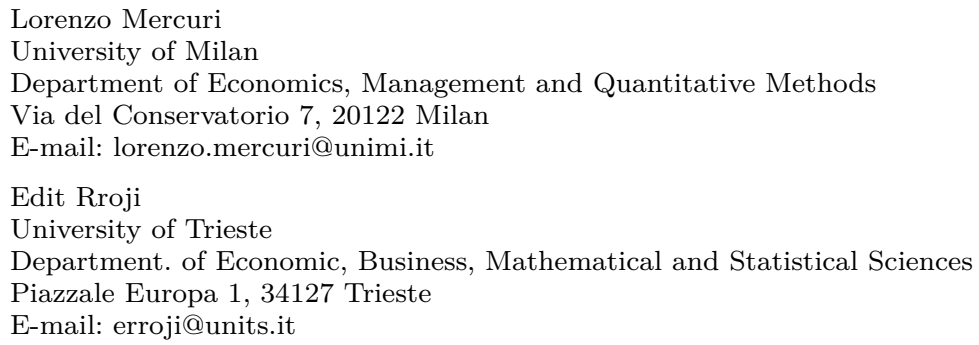


in financial literature. As shown in Rroji and Mercuri (2015), the MixedTS is more flexible in capturing higher moments and tail behavior varies from semi-heavy (i.e. the tail decays exponentially) to heavy (power law decay), while the tail behavior for the NVMM depends only on the tail behavior of the mixing random variable.

The first problem that we face in this paper is the selection of an equivalent martingale measure since we want to avoid arbitrage opportunities in option pricing. In particular, we consider two changes of measure that preserve the nature of the process: the Mean Correcting Martingale measure Schoutens (2003) and the Martingale Measure induced by the Esscher Transform Gerber et al. (1994).

The second issue that we analyze in this work is the extrapolation of the MixedTS parameters from quoted option prices. In literature, the standard approach is based on the choice of a specific loss function. Different kind of functions have been proposed and in general they can be classified in those in absolute terms (i.e. summation of the squared or absolute difference between theoretical and observed prices) or in relative terms. Both classes have advantages and problems like for instance a loss function in absolute terms gives higher weights to the errors referring to in the money options while the relative function overestimates out of the money options with short maturities (see Christoffersen and Jacobs, 2004, for a complete discussion). Alternative approaches try to see the calibration as a statistical procedure and consequently they provide standard errors associated to the estimated parameters. Generalized Method of Moments Arnold and Crack (1999), Bayesian option pricing Forbes et al. (2007) and Maximum Likelihood Estimation Hurn et al. (2014) belong to this class of methods. In this work we exploit the maximum likelihood estimation procedure and provide a unified methodology where the pricing error (i.e. the difference between quoted and theoretical price) follows a Generalized Normal distribution Johnson and Kotz (1970). The considered loss functions can be retrieved as special cases of the MLE approach considered. Moreover, the MLE provides the distribution of estimates and consequently a selection of models is possible using likelihood ratio tests or hypothesis testing for the parameters.

We introduce also a new $\mathrm{R}$ package called PricingMixedTS that allows the user to compute option prices when the underlying asset price follows an exponential MixedTS process. We provide routines for pricing based on the Mean Correcting Martingale measure and on the Esscher Transform. The presented calibration methodologies refer to different loss and likelihood functions.

The outline of the paper is as follows. In Section 2 we review the MixedTS distribution and discuss the process based on it. Option pricing for MixedTS process is developed in Section 3. In Section 4 we present calibration methodologies based on loss functions and on Maximum Likelihood Estimation. In Section 5 we explain the $\mathrm{R}$ package that implements the explained methodology and a numerical example is given in Section 6. Section 8 concludes the paper.

\section{Mixed Tempered Stable}

In this Section we review the Mixed Tempered Stable distribution (MixedTS hereafter) proposed by Rroji and Mercuri (2015) and its main properties. In particular we focus on the infinite divisibility property that is used for construction of the corresponding Lévy process. 
We say that a continuous random variable $Y$ follows a Mixed Tempered Stable distribution if:

$$
Y \stackrel{d}{=} \mu_{0}+\mu V+\sqrt{V} X
$$

where $X \mid V \sim \operatorname{stdCTS}\left(\alpha, \lambda_{+} \sqrt{V}, \lambda_{-} \sqrt{V}\right) . V$ is an infinitely divisible distribution defined on positive axis and its m.g.f always exists (see Rroji and Mercuri (2015)). The first four moments for the MixedTS are computed analytically. In this paper we focus on the case $V$ is Gamma distributed, i.e. $V \sim \Gamma\left(a, \sigma^{2}\right)$. It is also possible an alternatively parametrization for $V$ and for $Y$. Starting from the scale property of Gamma random variable:

$$
V=\sigma^{2} \tilde{V}
$$

where $\tilde{V} \sim \Gamma(a, 1)$. The MixedTS in (1) becomes:

$$
Y \stackrel{d}{=} \mu_{0}+\tilde{\mu} \tilde{V}+\sigma \sqrt{\tilde{V}} \tilde{X}
$$

with $\tilde{\mu}=\mu \sigma^{2}$ and $\tilde{X} \sim \operatorname{stdCTS}\left(\alpha, \lambda_{+} \sigma \sqrt{\tilde{V}}, \lambda_{-} \sigma \sqrt{\tilde{V}}\right)$, in this way we have similar parametrization of the Normal Variance Mean Mixtures.

Choosing $\mu=0$ we have the following special cases as shown in Rroji and Mercuri $(2015)^{1}$ :

- The Variance Gamma distribution is obtained choosing $\alpha=2$.

- The Standardized Classical Tempered Stable is obtained for $\sigma=\frac{1}{\sqrt{a}}$ and computing the limit for $a \rightarrow+\infty$.

- The Geometric Stable distribution for $\gamma>0$ is obtained by $\lambda_{+}=\lambda_{-}=\lambda, a=1$, $\sigma=\lambda^{\frac{\alpha-2}{\alpha}} \gamma^{\frac{\alpha}{2}} \sqrt{\mid \frac{\alpha(\alpha-1)}{\cos \left(\alpha \frac{\pi}{2}\right) \mid}}$ and computing the limit for $\lambda \rightarrow 0^{+}$.

The MixedTS r.v. is infinitely divisible and the characteristic function of r.v. $Y$ in (1) is given by:

$$
\Phi(u):=E\left[e^{i u Y}\right]=e^{i u \mu_{0}+\phi_{V}\left(i u \mu+L_{s t d C T S}\left(u, \alpha, \lambda_{+}, \lambda_{-}\right)\right)}
$$

where the $L_{\text {stdCTS }}\left(u, \alpha, \lambda_{+}, \lambda_{-}\right)$is the characteristic exponent of a Standardized Classical Tempered Stable:

$L_{s t d C T S}\left(u, \alpha, \lambda_{+}, \lambda_{-}\right)=\frac{\left(\lambda_{+}-i u\right)^{\alpha}-\lambda_{+}^{\alpha}+\left(\lambda_{-}+i u\right)^{\alpha}-\lambda_{-}^{\alpha}}{\alpha(\alpha-1)\left(\lambda_{+}^{\alpha-2}+\lambda_{-}^{\alpha-2}\right)}+\frac{i u\left(\lambda_{+}^{\alpha-1}-\lambda_{-}^{\alpha-1}\right)}{(\alpha-1)\left(\lambda_{+}^{\alpha-2}+\lambda_{-}^{\alpha-2}\right)}$.

The logarithm of m.g.f. for a Gamma r.v. with shape $a$ and scale $\sigma^{2}$ parameters $\phi_{V}(x)$ is defined as:

$$
\phi_{V}(x)=-a \ln \left(1-\sigma^{2} x\right) .
$$

Substituting formula (5) in (3) the MixedTS characteristic function becomes:

\footnotetext{
1 We refer the interested reader to Rroji and Mercuri (2015) for a more complete analysis on the shape of MixedTS distribution and on the behaviour of Skewness and Kurtosis for varying $\alpha$ and different combination of $\lambda_{+}$and $\lambda_{-}$
} 


$$
\Phi(u)=e^{i u \mu_{0}-a \ln \left[1-\sigma^{2}\left(i u \mu+L_{s t d C T S}\left(u, \alpha, \lambda_{+}, \lambda_{-}\right)\right)\right]} .
$$

It is worth noting that, since $V$ has an infinitely divisible distribution, the shape of the characteristic function in (3) identifies the distribution associated to a timechanged Lévy process [see Carr and Wu (2004b)] The time-changed Lévy process gives rise to an infinitely divisible distribution [see, theorems 7.10 and 30.1 in Sato (1999)]. Using the infinitely divisible property we have that:

$$
\{\Phi(X)\}^{t}=e^{i u \mu_{0} t+\phi_{V_{t}}\left(i u \mu+L_{s t d C T S}\left(u, \alpha, \lambda_{+}, \lambda_{-}\right)\right)}=\Phi\left(X_{t}\right)
$$

where $V_{t} \sim \Gamma\left(a t, \sigma^{2}\right)$ and $X_{t}$ is a $\operatorname{MixedTS}\left(\mu_{0} t, \mu, \sigma^{2}, a t, \alpha, \lambda_{+}, \lambda_{-}\right)$. In this way we are able to introduce a MixedTS Lévy process defined as follows:

Definition 1 Let $\left(\Omega, \mathcal{F},\left\{\mathcal{F}_{t}\right\}_{t>}, P\right)$ be a Filtered Probability Space, we define a MixedTS Lévy process $\left(X_{t}\right)_{t \geq 0}$ such that:

$-X_{0}=0$.

- The increments are independent and stationary.

$-X_{t-s}:=X_{t}-X_{s}$ is MixedTS distributed with parameters $\left(\mu_{0}(t-s), \mu, \sigma^{2}, a(t-s), \lambda_{+}, \lambda_{-}\right)$.

In Figure 1 and Figure 2 we show the sample paths of a MixedTS process for varying values of $\alpha$ parameters and different combination of parameters $\lambda_{+}, \lambda_{-}$. In particular we observe from Figure 2 that $\lambda_{+}$and $\lambda_{-}$controls the asymmetry in the distribution of the increments for each fixed lag. In particular, under $\mu=0$, the distributions are symmetric and the corresponding sample paths move around $\mu_{0}=0$. When $\lambda_{+}>\lambda_{-}$the distribution of increments is negatively skewed and, choosing $\mu_{0}=0$, the trend of sample paths seems to be negative while, if $\lambda_{+}<\lambda_{-}$ the trend seems to be increasing. In the Lévy process where the distribution of increments is a Normal Variance Mean Mixture, we have negative or positive skew if and only if $\mu$ is negative or positive.

In this paper we consider this new defined process for option pricing.

\section{Option Pricing Model}

In this Section we show how to price a European option under the assumption that the log returns of a risky asset are generated by a MixedTS Lévy process discussed in Section 2. In this case the market is incomplete and we decide to choose an Equivalent Martingale Measure using two approaches that preserve the nature of the process under the pricing measure (i.e. the log returns are again a MixedTS Lévy process under $Q$ measure): the Mean Correcting Martingale and the Esscher Transform.

Let $\left(\Omega,\left\{\mathcal{F}_{t}\right\}_{t \geq 0}, \mathbf{P}\right)$ be a Filtered Space, the price of a risky asset is:

$$
S_{t}=S_{0} e^{Y_{t}}, \quad t \geq 0 .
$$

where $Y_{t}$ is a MixedTS Lévy process with parameters $\left(\mu_{0}, \mu, \sigma, a, \lambda_{+}, \lambda_{-}\right)$.

The bank account dynamic is:

$$
B_{t}=B_{0} e^{r t}, \quad t \geq 0 .
$$



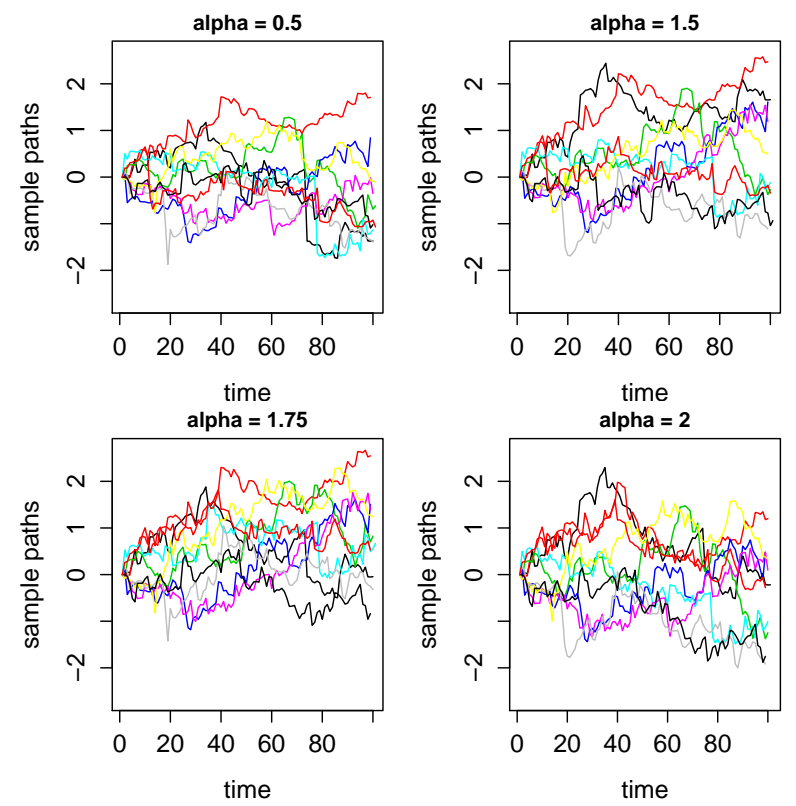

Fig. 1 Sample paths of MixedTS process with parameters $\mu_{0}=0, \mu=0, \sigma=0.2, \lambda_{+}=1$, $\lambda_{-}=1$ and varying $\alpha$
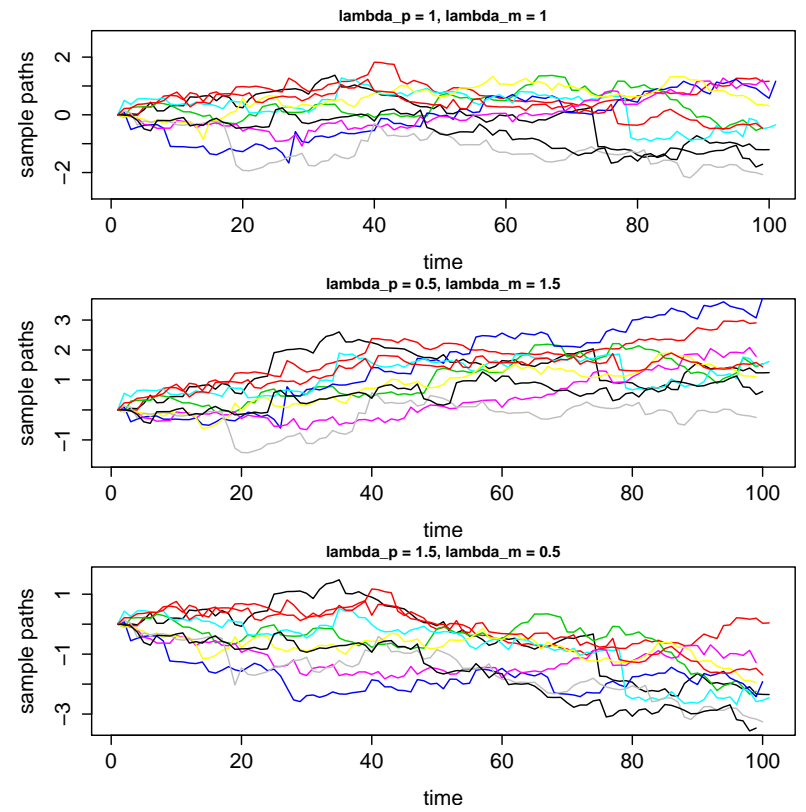

Fig. 2 Sample paths of MixedTS process with parameters $\mu_{0}=0, \mu=0, \sigma=0.2, \alpha=1.5$ and varying $\lambda_{+}$and $\lambda_{-}$ 
where the risk free rate $r$ is assumed to be constant for simplicity.

In order to select an equivalent martingale measure, in the following, we consider two different change of measures that preserve the nature of the process of the risky asset under the new measure: The Mean Correcting Martingale measure and the Esscher Transform based measure.

\subsection{Mean Correcting Martingale measure}

In this Section we use the Mean Correcting Martingale measure to determine an equivalent measure for pricing. The idea is to change the drift term such that the discounted price of the underlying asset is a martingale.

Let $Y_{t}$ to be a MixedTS process on a probability space $(\Omega, \mathcal{F}, P)$ where $P$ is the physical measure. For each $m \in \mathcal{R}$ we define a new process:

$$
Y_{t}^{m} \stackrel{d}{=} Y_{t}-m t
$$

and a new probability measure $Q^{m}$ defined on $(\Omega, \mathcal{F})$ that satisfies the following two properties:

i) $Q^{m} \sim P$ (i.e. $Q^{m}$ is equivalent to the physical measure $P$ ).

ii) $Q^{m}\left(Y_{t}^{m} \leq y\right):=P\left(Y_{t} \leq y\right) \quad \forall y \in \mathcal{R}$.

Fixed $t \leq T$, we choose $m$ such that:

$$
S_{t}=E^{Q}\left[e^{-r(T-t)} S_{T} \mid \mathcal{F}_{t}\right]=S_{t} e^{-r(T-t)} E^{Q}\left[e^{Y_{T-t}} \mid \mathcal{F}_{t}\right]
$$

Using the relation in (10) and the properties of the MixedTS process, equation (11) becomes:

$$
1=e^{-r(T-t)} E^{Q}\left[e^{Y_{T-t}^{m}+m(T-t)}\right]
$$

then

$$
e^{(r-m)(T-t)}=E^{Q}\left[e^{Y_{T-t}^{m}}\right]
$$

The property $i$ ) of the measure $Q^{m}$ implies that:

$$
e^{(r-m)(T-t)}=E^{P}\left[e^{Y_{T-t}}\right] .
$$

Applying the infinite divisibility property of the MixedTS, we have that:

$$
e^{(r-m)(T-t)}=E^{P}\left[e^{Y_{1}}\right]^{(T-t)} .
$$

The condition in (13) is well defined if $E^{P}\left[e^{Y_{1}}\right]<+\infty$ that means:

$$
1-\sigma^{2}\left[\mu+L_{s t d C T S}\left(-i, \alpha, \lambda_{+}, \lambda_{-}\right)\right]>0 .
$$

Solving equation (13) with respect to $m$, we determine $m_{*}$ as follows:

$$
m_{*}=r-\mu_{0}-\phi_{V}\left(\mu+L_{s t d C T S}\left(-i, \alpha, \lambda_{+}, \lambda_{-}\right)\right) .
$$

Log returns $X_{t}$, under the equivalent martingale measure, are generated from a MixedTS process defined in Section 2 with parameters $\left(\mu_{0}+m_{*}, \mu, \sigma^{2}, a, \alpha, \lambda_{+}, \lambda_{-}\right)$. 


\subsection{Esscher Transform}

An alternative approach for identifying a new martingale measure that preserves the nature of the process for the log returns (i.e log returns follow again a MixedTS after the change of measure) is the Esscher Transform introduced in option pricing literature by Gerber et al. (1994).

Under the assumption that $E\left(e^{c Y_{1}}\right)<+\infty$ in an open interval containing $c=0$, we are able to define for each $t$ a Radon Nikodym derivative as follows:

$$
\Lambda_{t}=\frac{e^{c Y_{t}}}{E\left[e^{c Y_{t}}\right]}
$$

The parameter $c$ is chosen in order to satisfy the martingale condition for the discounted price process:

$$
S_{0}=E^{Q}\left[e^{-\int_{0}^{t} r_{u} \mathrm{~d} u} S_{t} \mid \mathcal{F}_{0}\right] .
$$

Since $r$ is constant, the condition in (16) simplifies as follows:

$$
e^{r t}=E_{0}^{Q}\left[e^{Y_{t}}\right] .
$$

Using the Radon Nikodym derivative in (15), we obtain the Esscher equation:

$$
e^{r t}=E_{0}^{Q}\left[e^{Y_{t}}\right]=\frac{E_{0}^{P}\left[e^{(1+c) Y_{t}}\right]}{E_{0}^{P}\left[e^{c Y_{t}}\right]}=\frac{\Phi(-i(1+c))}{\Phi(-i c)} .
$$

Substituting the characteristic function of the MixedTS in (6), condition (17) becomes:

$$
e^{r t}=\frac{\Phi(-i(1+c))}{\Phi(-i c)}=\frac{e^{(1+c) \mu_{0} t+\phi_{V_{t}}\left((1+c) \mu+L_{s t d C T S}\left(-i(1+c), \alpha, \lambda_{+}, \lambda_{-}\right)\right)}}{e^{c \mu_{0} t+\phi_{V_{t}}\left(c \mu+L_{s t d C T S}\left(-i c, \alpha, \lambda_{+}, \lambda_{-}\right)\right)}}
$$

and after straightforward calculations:

$$
e^{\left(r-\mu_{0}\right)}=\frac{e^{\phi_{V}\left((1+c) \mu+L_{s t d C T S}\left(-i(1+c), \alpha, \lambda_{+}, \lambda_{-}\right)\right)}}{e^{\phi_{V}\left(c \mu+L_{s t d C T S}\left(-i c, \alpha, \lambda_{+}, \lambda_{-}\right)\right)}}
$$

where $V \sim \Gamma\left(a, \sigma^{2}\right)$.

It is worth to notice that equation (19) does not depend on $t$ and the solution $c$ is only a function of the model parameters. Using the Radon Nikodym derivative in (15), we are able to determine the moment generating function of the process at time $t$ as follows:

$$
E_{0}^{Q}\left[e^{\theta Y_{t}}\right]=E_{0}^{P}\left[\frac{e^{\left(\theta+c^{*}\right) Y_{t}}}{E_{0}^{p}\left[e^{c^{*} Y_{t}}\right]}\right] .
$$

Exploiting the characteristic function in (6), we have that:

$E_{0}^{Q}\left[e^{\theta Y_{t}}\right]=e^{t\left[\theta \mu_{0}+\phi_{V}\left(\left(\theta+c^{*}\right) \mu+L_{s t d C T S}\left(-i\left(\theta+c^{*}\right), \alpha, \lambda_{+}, \lambda_{-}\right)\right)-\phi_{V}\left(c^{*} \mu+L_{s t d C T S}\left(-i c^{*}, \alpha, \lambda_{+}, \lambda_{-}\right)\right)\right]}$.

First we analyze the term in $(21)$ : 
$\mathbf{A}:=\phi_{V}\left(\left(\theta+c^{*}\right) \mu+L_{s t d C T S}\left(-i\left(\theta+c^{*}\right), \alpha, \lambda_{+}, \lambda_{-}\right)\right)-\phi_{V}\left(c^{*} \mu+L_{s t d C T S}\left(-i c^{*}, \alpha, \lambda_{+}, \lambda_{-}\right)\right)$.

Since $V \sim \Gamma\left(a, \sigma^{2}\right)$, we have:

$$
\mathbf{A}=-a \ln \left[\frac{1-\sigma^{2}\left(\left(\theta+c^{*}\right) \mu+L_{s t d C T S}\left(-i\left(\theta+c^{*}\right), \alpha, \lambda_{+}, \lambda_{-}\right)\right)}{1-\sigma^{2}\left(c^{*} \mu+L_{s t d C T S}\left(-i c^{*}, \alpha, \lambda_{+}, \lambda_{-}\right)\right)}\right] .
$$

Adding and subtracting the term: $\sigma^{2} L_{s t d C T S}\left(-i c^{*}, \alpha, \lambda_{+}, \lambda_{-}\right)$in the numerator and we have:

$\mathbf{A}=-a \ln \left[1-\frac{\sigma^{2}\left(\theta \mu+L_{s t d C T S}\left(-i\left(\theta+c^{*}\right), \alpha, \lambda_{+}, \lambda_{-}\right)-L_{s t d C T S}\left(-i c^{*}, \alpha, \lambda_{+}, \lambda_{-}\right)\right)}{1-\sigma^{2}\left(c^{*} \mu+L_{s t d C T S}\left(-i c^{*}, \alpha, \lambda_{+}, \lambda_{-}\right)\right)}\right]$.

The quantity in (23), needs the evaluation of the term:

$$
\mathbf{B}:=L_{s t d C T S}\left(-i\left(\theta+c^{*}\right), \alpha, \lambda_{+}, \lambda_{-}\right)-L_{s t d C T S}\left(-i c^{*}, \alpha, \lambda_{+}, \lambda_{-}\right) .
$$

Using the characteristic exponent of a Standardized Tempered Stable in (4) we have:

$$
\begin{aligned}
\mathbf{B} & =\frac{\left(\lambda_{+}-c^{*}-\theta\right)^{\alpha}-\lambda_{+}^{\alpha}+\left(\lambda_{-}+c^{*}+\theta\right)^{\alpha}-\lambda_{-}^{\alpha}}{\alpha(\alpha-1)\left(\lambda_{+}^{\alpha-2}+\lambda_{-}^{\alpha-2}\right)}-\frac{\left(\lambda_{+}-c^{*}\right)^{\alpha}-\lambda_{+}^{\alpha}+\left(\lambda_{-}+c^{*}\right)^{\alpha}-\lambda_{-}^{\alpha}}{\alpha(\alpha-1)\left(\lambda_{+}^{\alpha-2}+\lambda_{-}^{\alpha-2}\right)} \\
& +\frac{\theta\left(\lambda_{+}^{\alpha-1}-\lambda_{-}^{\alpha-1}\right)}{(\alpha-1)\left(\lambda_{+}^{\alpha-2}+\lambda_{-}^{\alpha-2}\right)} \\
& =\frac{\left(\lambda_{+}-c^{*}-\theta\right)^{\alpha}-\left(\lambda_{+}-c^{*}\right)^{\alpha}+\left(\lambda_{-}+c^{*}+\theta\right)^{\alpha}-\left(\lambda_{-}+c^{*}\right)^{\alpha}}{\alpha(\alpha-1)\left(\lambda_{+}^{\alpha-2}+\lambda_{-}^{\alpha-2}\right)}+\frac{\theta\left(\lambda_{+}^{\alpha-1}-\lambda_{-}^{\alpha-1}\right)}{(\alpha-1)\left(\lambda_{+}^{\alpha-2}+\lambda_{-}^{\alpha-2}\right)} .
\end{aligned}
$$

Adding and subtracting the term: $\frac{\theta\left[\left(\lambda_{+}-c^{*}\right)^{\alpha-1}-\left(\lambda_{-}+c^{*}\right)^{\alpha-1}\right]}{(\alpha-1)\left[\left(\lambda_{+}-c^{*}\right)^{\alpha-2}+\left(\lambda_{-}+c^{*}\right)^{\alpha-2}\right]} \frac{\left[\left(\lambda_{+}-c^{*}\right)^{\alpha-2}+\left(\lambda_{-}+c^{*}\right)^{\alpha-2}\right]}{\left[\lambda_{+}^{\alpha-2}+\lambda_{-}^{\alpha-2}\right]}$, we obtain:

$$
\begin{aligned}
\mathbf{B} & =L_{s t d C T S}\left(-i \theta, \alpha, \lambda_{+}-c^{*}, \lambda_{-}+c^{*}\right) \frac{\left[\left(\lambda_{+}-c^{*}\right)^{\alpha-2}+\left(\lambda_{-}+c^{*}\right)^{\alpha-2}\right]}{\left[\lambda_{+}^{\alpha-2}+\lambda_{-}^{\alpha-2}\right]} \\
& +\theta \frac{\left[\lambda_{+}^{\alpha-1}-\left(\lambda_{+}-c^{*}\right)^{\alpha-1}+\left(\lambda_{-}+c^{*}\right)^{\alpha-1}-\lambda_{-}^{\alpha-1}\right]}{(\alpha-1)\left(\lambda_{+}^{\alpha-2}+\lambda_{-}^{\alpha-2}\right)} .
\end{aligned}
$$

Substituting term (25) in (23), we have:

$$
\begin{aligned}
\mathbf{A} & =-a \ln \left[1-\frac{\sigma^{2}\left[\left(\lambda_{+}-c^{*}\right)^{\alpha-2}+\left(\lambda_{-}+c^{*}\right)^{\alpha-2}\right]}{\left[1-\sigma^{2}\left(c^{*} \mu+L_{\text {stdCTS }}\left(-i c^{*}, \alpha, \lambda_{+}, \lambda_{-}\right)\right)\right]\left[\lambda_{+}^{\alpha-2}+\lambda_{-}^{\alpha-2}\right]}\right. \\
& *\left[\theta\left(\mu+\frac{\left[\lambda_{+}^{\alpha-1}-\left(\lambda_{+}-c^{*}\right)^{\alpha-1}+\left(\lambda_{-}+c^{*}\right)^{\alpha-1}-\lambda_{-}^{\alpha-1}\right]}{(\alpha-1)\left(\lambda_{+}^{\alpha-2}+\lambda_{-}^{\alpha-2}\right)}\right) \frac{\left[\lambda_{+}^{\alpha-2}+\lambda_{-}^{\alpha-2}\right]}{\left[\left(\lambda_{+}-c^{*}\right)^{\alpha-2}+\left(\lambda_{-}+c^{*}\right)^{\alpha-2}\right]}\right. \\
& \left.\left.+L_{\text {stdCTS }}\left(-i \theta, \alpha, \lambda_{+}-c^{*}, \lambda_{-}+c^{*}\right)\right]\right] .
\end{aligned}
$$

Defining: 


$$
\left\{\begin{array}{l}
\hat{\mu}=\left(\mu+\frac{\left[\lambda_{+}^{\alpha-1}-\left(\lambda_{+}-c^{*}\right)^{\alpha-1}+\left(\lambda_{-}+c^{*}\right)^{\alpha-1}-\lambda_{-}^{\alpha-1}\right]}{(\alpha-1)\left(\lambda_{+}^{\alpha-2}+\lambda_{-}^{\alpha-2}\right)}\right) \frac{\left[\lambda_{+}^{\alpha-2}+\lambda_{-}^{\alpha-2}\right]}{\left[\left(\lambda_{+}-c^{*}\right)^{\alpha-2}+\left(\lambda_{-}+c^{*}\right)^{\alpha-2}\right]} \\
\hat{\sigma}^{2}=\frac{\sigma^{2}\left[\left(\lambda_{+}-c^{*}\right)^{\alpha-2}+\left(\lambda_{-}+c^{*}\right)^{\alpha-2}\right]}{\left[1-\sigma^{2}\left(c^{*} \mu+L_{s t d C T S}\left(-i c^{*}, \alpha, \lambda_{+}, \lambda_{-}\right)\right)\right]\left[\lambda_{+}^{\alpha-2}+\lambda_{-}^{\alpha-2}\right]} \\
\hat{\lambda}_{+}=\lambda_{+}-c^{*} \\
\hat{\lambda}_{-}=\lambda_{-}+c^{*}
\end{array}\right.
$$

with $c^{*} \in\left(\lambda_{-}, \lambda_{+}\right)$, under the Equivalent Martingale Measure induced by the Esscher Transform, the log returns follow a MixedTS process with parameters $\left(\mu_{0}, \hat{\mu}, \hat{\sigma}^{2}, a, \alpha, \hat{\lambda}_{+}, \hat{\lambda}_{-}\right)$.

We conclude this section observing that Mean Correcting Martingale and Esscher Transform return, under the pricing measure, a MixedTS process for log-price. In both case the characteristic function is obtained in a closed form formula and the option call prices can be evaluated using any pricing formula based on the characteristic function. In this work we use the same approach developed in Carr and Madan (1999) that allow us to apply the Fast Fourier Transform algorithm. In our case the dumping parameter ${ }^{2}$ is fixed to 0.75 as suggested in Schoutens et al. (2004).

\section{Calibration Procedure}

The aim of this Section is to describe how to estimate model parameters using the quoted option prices. The classical approach is based on standard calibration methods. The parameters are obtained by minimizing a distance between theoretical and empirical prices and different measures of fit have been developed such as:

- Root Mean Squared Error (RMSE)

$$
\mathrm{RMSE}=\sqrt{\sum_{i=1}^{N} \frac{\left(C_{i}^{\text {mkt }}-C_{i}^{\text {theo }}\right)^{2}}{N}} .
$$

- Root Mean Squared Percentage Error (RMSPE)

$$
\mathrm{RMSPE}=\sqrt{\frac{1}{N} \sum_{i=1}^{N}\left(\frac{C_{i}^{m k t}-C_{i}^{\text {theo }}}{C_{i}^{m k t}}\right)^{2}} .
$$

- Average Absolute Error (AAE)

$$
\mathrm{AAE}=\sum_{i=1}^{N} \frac{\left|C_{i}^{\text {mkt }}-C_{i}^{\text {theo }}\right|}{N} .
$$

- Average Relative Percentage Error (ARPE)

$$
\mathrm{ARPE}=\frac{1}{N} \sum_{i=1}^{N} \frac{\left|C_{i}^{m k t}-C_{i}^{\text {theo }}\right|}{C_{i}^{\text {mkt }}} .
$$

2 In the PricingMixedTS the dumping parameters can be selected by the user, the default value is 0.75 
A detailed discussion on the properties of the different objective functions can be found in Christoffersen and Jacobs (2004) and in Schoutens (2003). It is worth to notice that minimizing the above distances gives us only one value for each parameter. Nevertheless, calibration is a statistical methodology and consequently we need to determine also an appropriate confidence interval for the estimate. In literature three different statistical estimation procedures have been considered: Generalized Methods of Moments (see Arnold and Crack (1999) for a discussion), a Bayesian option pricing (see Forbes et al. (2007)) and a Maximum Likelihood Estimation procedure (see Hurn et al. (2014) and references therein).

In this work, we consider the Maximum Likelihood Estimation procedure. We assume market option prices to be composed by two elements: model option prices and a white noise error. Following Hurn et al. (2014), the pricing error can be additive or multiplicative, that means, in the first case the difference between observed and theoretical option price is a zero mean gaussian random variable while, in the second case, the difference between the logarithm of the theoretical and observed price is a zero mean gaussian random variable. We report four types of pricing errors where the first two are additive and remaining are multiplicative. Hereafter, we indicate with $\theta$ the vector containing the model parameters, i.e. $\theta:=\left(\mu_{0}, \mu, \sigma, a, \alpha, \lambda_{+}, \lambda_{-}\right)$.

- Additive Pricing Error (APE). The pricing error is a zero mean Gaussian r.v. where the standard error is the same for each option available in the market:

$$
C_{i}^{\text {mkt }}=C_{i}^{\text {theo }}+\epsilon, \epsilon \sim N\left(0, \sigma_{\epsilon}^{2}\right) \forall i=1, \ldots, N .
$$

- Additive Proportional Pricing Error (APPE). The observed price is obtained as a sum of the theoretical price with a zero mean gaussian r.v. where the standard error is proportional to the market price:

$$
C_{i}^{m k t}=C_{i}^{\text {theo }}+\epsilon_{i}, \epsilon_{i} \sim N\left(0, \sigma_{\epsilon}^{2}\left(C_{i}^{m k t}\right)^{2}\right) \forall i=1, \ldots, N .
$$

- Multiplicative Pricing Error 1 (MPE1). The difference between log market price and log theoretical price is a zero mean Gaussian r.v. with constant variance $\sigma^{2}$ :

$$
\ln \left(C_{i}^{m k t}\right)=\ln \left(C_{i}^{o b s}\right)+\epsilon, \epsilon \sim N\left(0, \sigma_{\epsilon}^{2}\right) \forall i=1, \ldots, N .
$$

- Multiplicative Pricing Error 2 (MPE2) The multiplicative error is chosen in order to ensure the condition $E\left(C_{i}^{m k t}\right)=C_{i}^{\text {theo }}$, i.e. we have:

$$
C_{i}^{m k t}=C_{i}^{\text {theo }} e^{\epsilon}, \epsilon \sim N\left(-\frac{1}{2} \sigma_{\epsilon}^{2}, \sigma_{\epsilon}^{2}\right) \forall i=1, \ldots, N
$$

It is worth to notice that the RMSE and APE approaches lead to the same estimates for the model parameters. Indeed, the loglikelihood in the APE approach is equal to:

$$
\operatorname{LogLik}_{A P E}\left(\theta, \sigma_{\epsilon}^{2}\right)=-\frac{N}{2} \ln (2 \pi)-\frac{N}{2} \ln \left(\sigma_{\epsilon}^{2}\right)-\frac{1}{2 \sigma_{\epsilon}^{2}} \sum_{i=1}^{N}\left(C_{i}^{m k t}-C_{i}^{t h e o}\right)^{2} .
$$

The maximization of (36) can be splitted in two problems. Indeed, the contribution of the model parameters $\theta$ is determined by minimization of the RMSE. Once the 
optimal value for $\theta$ is obtained, we maximize the quantity in (36) with respect to $\sigma_{\epsilon}^{2}$ and get:

$$
\frac{\partial \log \operatorname{Lik}_{A P E}\left(\theta^{*}, \sigma_{\epsilon}^{2}\right)}{\partial \sigma_{\epsilon}^{2}}=-\frac{N}{2 \sigma_{\epsilon}^{2}}+\frac{1}{2\left(\sigma_{\epsilon}^{2}\right)^{2}} \sum_{i=1}^{N}\left(C_{i}^{m k t}-C_{i}^{t h e o}\right)^{2}=0
$$

and finally:

$$
\sigma_{\epsilon}^{2}=\frac{\sum_{i=1}^{N}\left(C_{i}^{m k t}-C_{i}^{t h e o}\right)^{2}}{N} \geq 0 .
$$

The same arguments hold for the relation between RMSPE and APPE. In this case the estimate of $\sigma_{\epsilon}^{2}$ is:

$$
\sigma_{\epsilon}^{2}=\frac{1}{N} \sum_{i=1}^{N}\left(\frac{C_{i}^{m k t}-C_{i}^{\text {theo }}}{C_{i}^{m k t}}\right)^{2} \geq 0
$$

We show a generalized calibration procedure that tries to combine the previous methods (except for MPE1 and MPE2). The idea behind the proposed approach is that the error term is a Generalized Normal random variable Johnson and Kotz (1970). A continuous random variable $X$ is a Generalized Normal r.v. with parameters $(\eta, \gamma, \beta)$ if its density function is given by:

$$
f_{X}(x)=\frac{\beta}{2 \gamma \Gamma(1 / \beta)} e^{-(|x-\eta| / \gamma)^{\beta}}
$$

where the position parameter $\eta$ belongs to the real line, the scale parameter $\gamma$ and the shape parameter $\beta$ assumes non negative values. This random variable has been widely used in Bayesian analysis and robustness see Box and Tiao (1962); Subbotin (1923); Tiao and Lund (1970) for instance. In the following, we use the notation $X \sim$ $\operatorname{GND}(\eta, \gamma, \beta)$ for indicating that that the distribution of a r.v. $X$ is a Generalized Normal with parameters $(\eta, \gamma, \beta)$.

The shape parameter $\beta$ manages the tails' behavior. In particular, if $\beta>2$ the tails are thinner than those of a Normal distribution, while for $\beta \in(0,2)$ the tails are heavier. The distribution has the following special cases:

- For $\beta=2$, we obtain a normal random variable with mean $\gamma$ and variance $\frac{\gamma^{2}}{2}$.

- For $\beta=1$, the double exponential r.v. is retrieved.

- For $\beta \rightarrow \infty$, the Generalized Normal converges to the Uniform distribution defined on $(\eta-\gamma, \eta+\gamma)$.

These properties make this distribution appealing for building a unified calibration procedure based on the likelihood function of pricing error. In this paper, we consider three nested different procedures: Additive Generalized Normal Error (AGNE), Additive Proportional Generalized Normal Error (APGNE) and Additive Mixed Generalized Normal Error (AMGNE).

- In the Additive Generalized Normal Error (AGNE) we assume market prices to be defined as:

$$
C_{i}^{m k t}=C_{i}^{\text {theo }}+\epsilon_{i}, \epsilon_{i} \sim G N D(0, \gamma, \beta) i=1, \ldots, N
$$


where the error terms $\epsilon_{i}$ are i.i.d Generalized Normal r.v.'s, the loglikelihood function of the error term is obtained as follows:

$$
\operatorname{LogLik}_{A G N E}(\theta, \gamma, \beta)=N \ln (\beta)-N \ln (2 \gamma \Gamma(1 / \beta))-\sum_{i=1}^{N}\left(\frac{\left|C_{i}^{\text {mkt }}-C_{i}^{\text {theo }}\right|}{\gamma}\right)^{\beta} \text {. }
$$

It is worth to observe that the value of shape parameter $\beta$ allows the user to choose between the RMSE and the AAE procedures analyzed previously. Indeed, for $\beta=1$ the model parameters are obtained by maximizing the loglikelihood in (40) that is equivalent to the minimization of the AAE measure while, for $\beta=2$, we have the same result of minimizing the RMSE quantity.

- In the Additive Proportional Generalized Normal Error (APGNE), the market price is given as:

$$
C_{i}^{m k t}=C_{i}^{\text {theo }}+\epsilon_{i}, \epsilon_{i} \sim G N D\left(0, \gamma C_{i}^{m k t}, \beta\right) i=1, \ldots, N
$$

where the error terms $\epsilon_{i}$ are independent Generalized Normal r.v.'s. In this case, it is possible to obtain the RMSPE (choosing $\beta=2$ ) and the APPE (choosing $\beta=2$ ) as special cases. The loglikelihood is computed as follows:

$$
\begin{aligned}
\operatorname{LogLik}_{A P G N E}(\theta, \gamma, \beta) & =N \ln (\beta)-N \ln (2 \gamma \Gamma(1 / \beta))-\sum_{i=1}^{N} \ln \left(C_{i}^{m k t}\right) \\
& -\sum_{i=1}^{N}\left(\frac{\left|C_{i}^{m k t}-C_{i}^{t h e o}\right|}{\gamma C_{i}^{\text {mkt }}}\right)^{\beta} .
\end{aligned}
$$

- In the Additive Mixed Generalized Normal Error (AMGNE), we assume market price to be:

$$
C_{i}^{m k t}=C_{i}^{\text {theo }}+\epsilon_{i}, \epsilon_{i} \sim G N D\left(0, \gamma\left(C_{i}^{m k t}\right)^{\rho}, \beta\right) i=1, \ldots, N
$$

where $\rho \in[0,1]$.

AMGNE is the most general estimation procedure analyzed in this Section. Indeed, the AMGNE encompasses the APGNE and the APGNE introduced above. If $\rho=0$, we have the AGNE approach where $\rho=1$ corresponds to the APGNE method. In this case the loglikelihood has the following form:

$$
\begin{aligned}
\operatorname{LogLik}_{A M G N E}(\theta, \gamma, \beta) & =N \ln (\beta)-N \ln (2 \gamma \Gamma(1 / \beta))-\sum_{i=1}^{N} \ln \left[\left(C_{i}^{m k t}\right)^{\rho}\right] \\
& -\sum_{i=1}^{N}\left(\frac{\left|C_{i}^{\text {mkt }}-C_{i}^{\text {theo }}\right|}{\gamma\left(C_{i}^{m k t}\right)^{\rho}}\right)^{\beta} .
\end{aligned}
$$

It is worth to notice that the method coincides with the ARPE when $\beta=1$ and $\rho=1$. The RMSPE is obtained if $\beta=1$ and $\rho=1$ while the AAE for $\beta=1$ and $\rho=0$ and the RMSE for $\beta=2$ and $\rho=0$.

A general approach like the AMGNE is a tool for comparing and correctly choosing among different estimation methodologies based on likelihood ratio tests or through hypothesis testing for parameters $\beta$ and $\rho$. In our approach no ambiguity in calibration method choice remains. 


\section{R package PricingMixedTS}

In this Section, we describe the $\mathrm{R}$ package PricingMixedTS that contains several routines for option pricing under the assumption that log returns follow a MixedTS process. This package is written using the $\mathrm{S} 4$ object oriented programming language and is freely downloadable from https://r-forge.r-project.org/projects/pricingmixedts. Once the package is installed, the user can call it using the comand library as done below:

library("PricingMixedTS")

\subsection{Classes}

Three classes are used: param.MixedTS, OptionData and Calibrates.

An object of class param. MixedTS identifies the MixedTS parameters and other information related to the MixedTS distribution. This object is constructed by the user through function setMixedTS.param. Its slots are:

- @mu0: a numeric object for the constant term $\mu_{0}$.

- @mu: a numeric object for the parameter $\mu$.

- Qsigma: a numeric object. In this case negative values for $\sigma$ are not allowed.

- @a: a numeric object. This slot is filled if the mixing density is Gamma distributed and corresponds to its shape parameter.

- Qalpha: a numeric object that takes value from 0 to 2 . If $\alpha=2$, the Mixed Tempered Stable becomes the Normal Variance Mean Mixture.

- @lambda_p: a positive numeric object. It is the right tempering parameter of the standardized classical Tempered Stable used for building the MixedTS r.v.

- Qlambda_m: a positive numeric object. It is the left tempering parameter of the standardized Classical Tempered Stable used for building the MixedTS.

- @Mixing: a string object indicating the nature of the mixing density $V$. If Mixing = "Gamma" (default value) the mixing r.v. is Gamma distributed. If Mixing = "User", the user should specify the log of the moment generating function of the random variable $V$.

- QparamMixing: a list object. It is an empty list if the slot Mixing = "Gamma" otherwise it is used to pass the values of the Mixing density parameters defined by the user.

- @MixingLogMGF: this slot contains a function that returns the logarithm of mgf for the mixing density.

- @Parametrization: a string that indicates the parametrization of the MixedTS r.v. used. In particular if Parametrization = "A" (default value) we use the definition in (1) otherwise the parametrization in (2). In the last case the user chooses Parametrization = "B"

OptionData. An object of this class contains all the information about options available in the market. This object is constructed by function DataOpt. The slots that compose an object of class OptionData are listed below:

- @UnderPrice: a numeric object that represents the current underlying asset price.

- QPrice0pt: a numeric object that is the current option price. 
- @ImpliedVol: a numeric object that is the corresponding Black and Scholes implied volatility.

- @TimeToMat: a numeric object indicating the time to maturity of the option.

- QStrike: a numeric object that represents the strike price of the option

- QType: a character object. If the Type $=\mathrm{Call}$ we consider an European call option while with Type = Put we have an European put option is

- Orate: a numeric object that is the risk free rate.

- Qqyield: a numeric object that contains the dividend yield of the underlying asset. If the underlying asset is a commodity, the slot contains the convenience yield.

- Qdateobs: a data object indicating the time where we observe the option prices.

Calibrates An object of this class contains the parameters of an exponential MixedTS process obtained from the option prices available in the market. This object is built internally through the function calibrate and inherits slots and methods from param. MixedTS. Moreover, an object of this class has the following additional slots containing the results of the estimation procedure.

- @coef: a numeric object that contains the calibrated parameters of the model.

- Qvcov: a matrix object that is the variance-covariance matrix of estimated parameters.

- @obj: a numeric object that represents the value of the distance measure in the estimation procedure.

\subsection{Methods}

The method Qparam.MixedTS MixedTS converts the parameters of an exponential MixedTS process under the real measure into the corresponding parameters under the equivalent martingale measure obtained by the Esscher Transform or the Mean Correcting Martingale measure. The inputs are: object, ret and type.

The argument object is an object of class param. MixedTS and contains information about the process under the physical measure. The risk free rate is passed through the input ret and, since the market is incomplete, the user is allowed to choose an equivalent martingale measure using the Esscher Transform or the Mean Correcting Martingale measure by setting QMeasure equal to FALSE or TRUE respectively.

The method OptionPrice computes option prices with the arguments:

- object: an object of class param. MixedTS that contains all information about the log return process.

- S0: a numeric object of the current underlying asset price.

- Strike: a numeric object that represents the level of the strike price.

- TimeToMat: a numeric object that represents the time to expiry for options.

- ret: a numeric object that contains the constant risk free rate.

- basis: a numeric object indicating the number of days in one year. The default value is 360 .

- Qmeasure: a logical variable. If Qmeasure = FALSE the equivalent martingale is selected according to the Esscher Transform otherwise the Mean Correcting Martingale measure is used when Qmeasure = TRUE.

- type: a character object that indicates whether we are working with a call (type $=$ CALL) or with a put (type = CALL). 
The method OptionPrice is also used internally by the function GenerOptMrk that allows the user to build a simulated option market since it is possible to adding an error term through the additional input error.

The method calibrate estimates the MixedTS parameters using quoted option prices. All the approaches illustrated in Section 4 are available. The arguments are: model, Data, Qmeasure, basis and method. Qmeasure, basis are the same inputs as for method OptionPrice. The remaining quantities are: where the arguments mean:

- model: an object of class param.MixedTS that contains the starting values for the model parameters in the optimization routine.

- Data: an object of class OptionData that contains the quoted option prices.

- method: a character object that is used for specifying the estimation procedure considered the choices are:

- method $=$ "RMSE" for the Mean Squared Error.

- method = "RMSPE" for the Mean Squared Percentage Error.

- method = "AAE" for the Average Absolute Error.

- method = "ARPE" for the Average Relative Percentage Error.

- method $=$ "APPE" for the Additive Proportional Pricing Error.

- method = "APE" for the Additive Pricing Error.

- method = "MPE1" for the Multiplicative Pricing Error 1.

- method = "MPE2" for the Multiplicative Pricing Error 2.

- method = "AGNE" for the Additive Generalized Normal Error.

- method = "APGNE" for the Additive Proportional Generalized Normal Error.

- method = "AMGNE" for the Additive Mixed Generalized Normal Error.

\section{Numerical Examples}

In this Section we show how to use the PricingMixedTS package in the estimation of model parameters using option prices. To verify the estimation ability of the different approaches analyzed in Section 4, we consider a simulated market where option prices are computed as a summation of two components: model prices and a zero mean gaussian noise. The model is estimated under the real measure and the equivalent martingale measure is selected based on the Esscher Transform.

First, we define the model under the real measure using the constructor setMixedTS . param.

Modpar <- setMixedTS. $\operatorname{param}(\mathrm{mu} 0=0, \mathrm{mu}=-0.01$, sigma $=0.10$,

$\mathrm{a}=1.67$, alpha $=1.7$, lambda_p $=1.1$, lambda_m $=1.05$ )

We define the contract features:

TimeToMat $<-c(30,45,60)$

Strike <- c(97.5, 98, 99, 100, 101, 102, 102.5)

In order to generate the option prices, we use the function GenerOptMrk that internally calls the OptionPrice method:

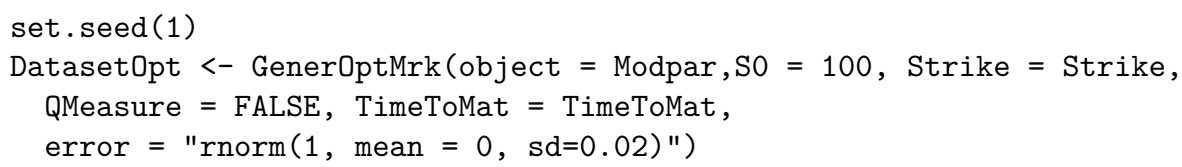


The function allows the user to insert an additive noise in the prices using the argument error.

The simulated prices are reported in Figure 3. We observe that, even adding a gaussian noise, the value of standard error is small enough to ensure the standard behavior of prices, that means, decreasing convex function w.r.t strike and increasing function w.r.t time to maturity.

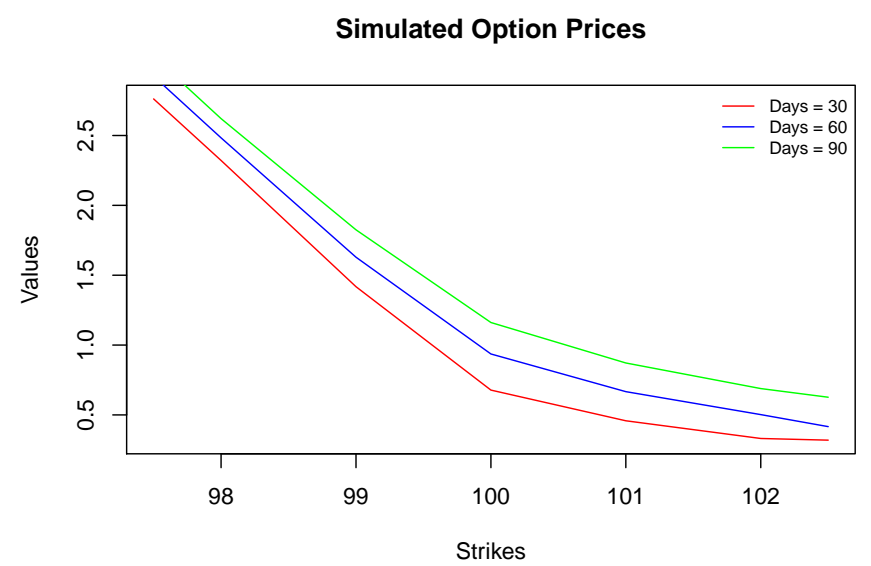

Fig. 3 Option price behavior for varying strikes and time to maturities.

In the estimation procedure, we consider only methods that are coherent with an additive noise, i.e. RMSE, APE, AGNE and ANGNE. Command lines for estimation are reported below:

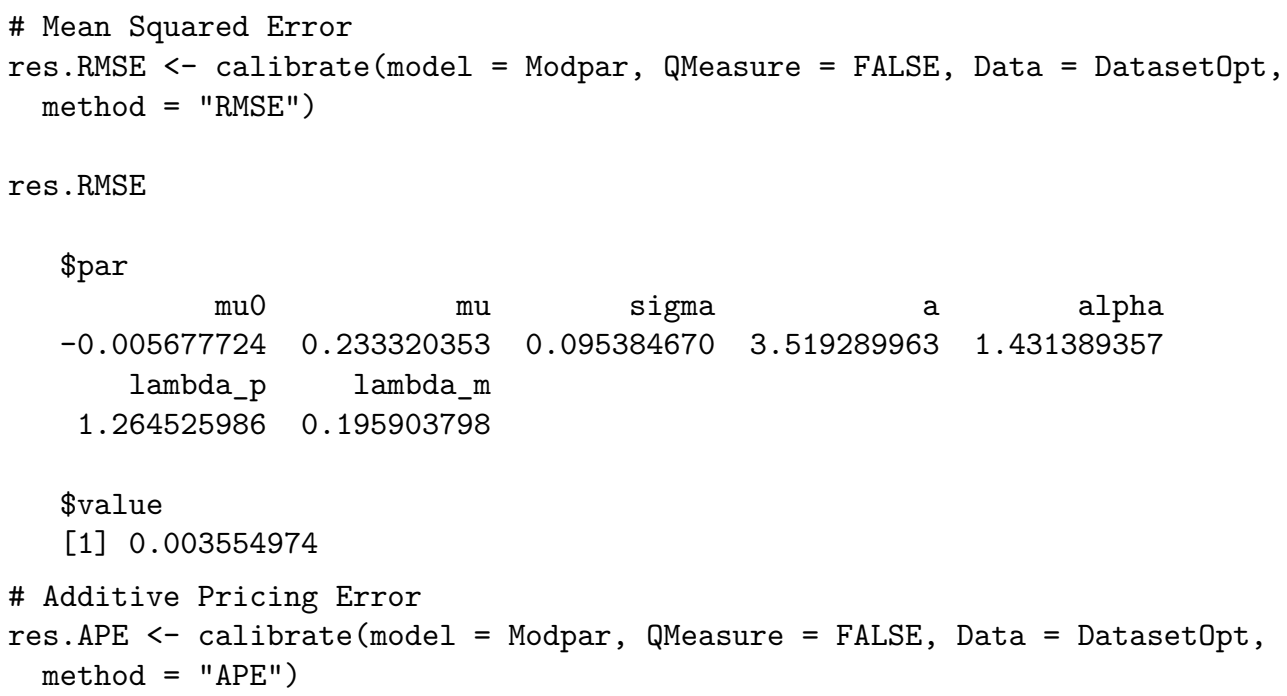

In order to show the result, we use the summary method for estimates and their standard error while the method logLik computes the level of the loglikelihood. 
summary (res. APE)

\#\# Maximum likelihood estimation

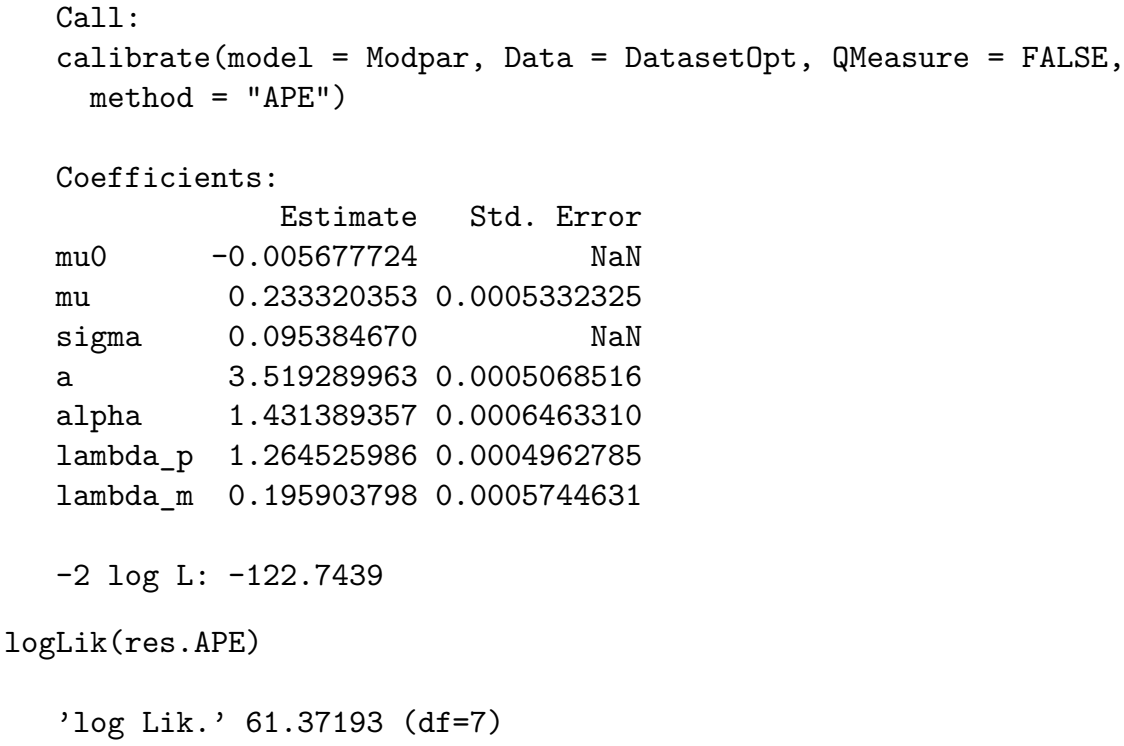

In the following we report the command lines for the other methods:

\# Additive Generalized Normal Error

res.AGNE <- calibrate (model = Modpar, QMeasure = FALSE, Data = DatasetOpt, method = "AGNE")

summary (res. AGNE)

\#\# Maximum likelihood estimation

Call:

calibrate $($ model $=$ Modpar, Data $=$ DatasetOpt, QMeasure $=$ FALSE, method = "AGNE")

Coefficients:

Estimate Std. Error

muo $\quad-0.0088167030 .0005179283$

$\mathrm{mu} \quad-0.182573728 \quad 0.0014803146$

sigma $\quad 0.0815120370 .0002618718$

a $\quad 1.8789378530 .0013934064$

alpha $\quad 1.8060590200 .0017444467$

lambda_p $\quad 2.4648504690 .0033470183$

lambda_m $\quad 1.0443494630 .0021938962$

beta_err 0.5197695430 .0459639251

gamma_err 0.0020740950 .0001997059 
$-2 \log \mathrm{L}:-125.4714$

$\operatorname{logLik}($ res.AGNE)

'log Lik.' $62.73572(\mathrm{df}=9)$

Due to the the complex form of the loglikelihood function in the ANGNE approach, it is needed to choose an appropriate starting value in the optimization routine. In the following we decide to initialize the procedure using the estimates obtained with the APE method where the $\mu_{0}$ is multiplied by 0.9 :

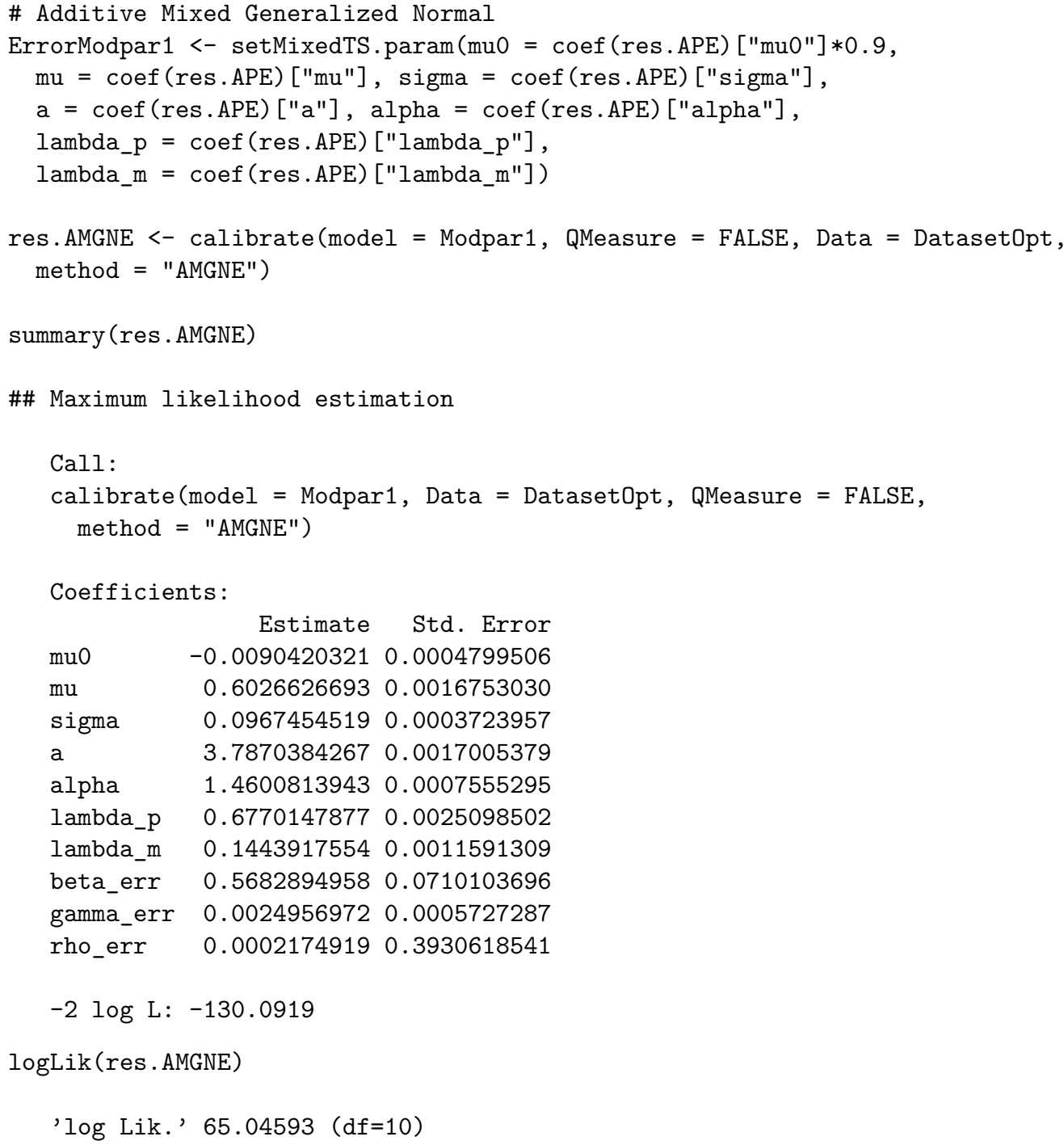

As expected, the best performance in term of loglikelihood is achieved using the AMGNE method. This result is not surprising since, as observed in Section 4, this 
approach has the others as special cases. Nevertheless, the possibility of computing the loglikelihood allows us to select an approach using the likelihood ratio test. In particular, the value of the ratio test between the AMGNE and the APE approaches is 7.3480 (that corresponds to a p value 0.0616 ) that means, the restricted method (APE) is more suitable.

We show how to determine the distribution of the option price with the APE method. Once the parameters are estimated, we compute the option price using OptionPrice method. We consider a European call option with time to maturity 30 days, strike price 95 and underlying asset price 100 .

To extrapolate the estimated parameters from the object res.APE we use the method coef:

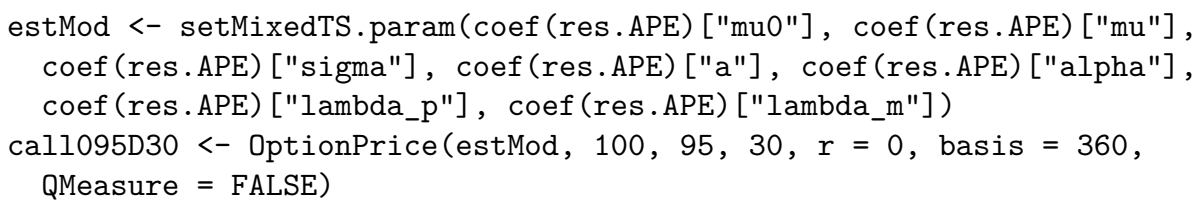

We determine the grid of strikes and use the dnorm function to determine the density of the option price. The standard deviation of the error term is stored in the slot fullcoef:
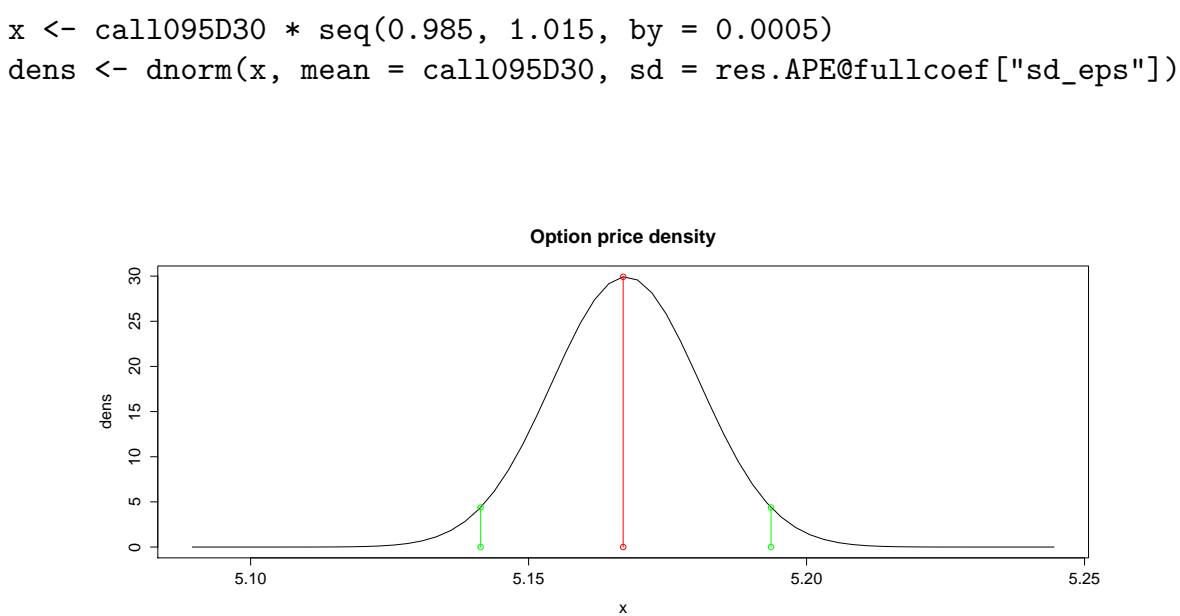

Fig. 4 Distribution of the option price when the underlying price is 100 , strike price 95 and maturity 30 days.

Figure 4 shows the density of the option price with maturity 30 days and strike 95 based on the estimated parameters. The red line corresponds to the theoretical price and the area between the green lines is the probability of the event $\left|\frac{\text { Call }^{m k t}-\text { call }^{\text {theo }}}{\sigma_{\epsilon}}\right| \leq 1.96$ ( $\sigma_{\epsilon}$ is the standard deviation of the pricing error term). In conclusion, if the MixedTS is a correct model and the estimation procedure is the $\mathrm{APE}$, we expect that the real option price to belong to the interval $\left[\right.$ Call $^{\text {theo }}-1.96 \sigma_{\epsilon}$, Call $\left.^{\text {theo }}+1.96 \sigma_{\epsilon}\right]$. 


\section{Sensitivity and real data analysis}

This Section is composed by two parts. The first one is devoted to a sensitivity analysis with respect to the $\alpha$ parameters. In the second part we study the behaviour of the MixedTS model on real option prices and compare the calibration performance with Variance Gamma model. As observed in Section 3, the Variance Gamma model is obtained as a special case of a MixedTS when $\alpha=2$.

In order to study the behaviour of option prices when $\alpha$ varies, we consider, under real measure, three models that are identified by the following parameters $\mu_{0}=0$, $\mu=0, \sigma=1, a=1$,alpha $=(0.8,1.25,1.65) \lambda_{p}=1.1$ and $l a m b d a_{m}=0.9$ on monthly basis.

In table 1 we report the corresponding parameters obtained through Esscher transform and the Mean Correcting Martingale approach for each model.

Table 1 Model Parameters under real and equivalent martingale measures

\begin{tabular}{|c|c|c|c|c|c|c|c|}
\hline \multicolumn{8}{|c|}{ Model 1} \\
\hline & $\mu_{0}$ & $\mu$ & $\sigma$ & $a$ & $\alpha$ & $\lambda_{p}$ & $\lambda_{m}$ \\
\hline Real & 0 & 0 & 0.150 & 2.500 & 0.800 & 1 & 1.500 \\
\hline $\mathrm{MCM}$ & -0.067 & 0 & 0.150 & 2.500 & 0.800 & 1 & 1.500 \\
\hline Essch & 0 & -0.632 & 0.165 & 2.500 & 0.800 & 1.750 & 0.750 \\
\hline \multicolumn{8}{|c|}{ Model 2} \\
\hline & $\mu_{0}$ & $\mu$ & $\sigma$ & $a$ & $\alpha$ & $\lambda_{p}$ & $\lambda_{m}$ \\
\hline Real & 0 & 0 & 0.150 & 2.500 & 1.250 & 1 & 1.500 \\
\hline $\mathrm{MCM}$ & -0.051 & 0 & 0.150 & 2.500 & 1.250 & 1 & 1.500 \\
\hline Essch & 0 & -0.688 & 0.158 & 2.500 & 1.250 & 1.750 & 0.750 \\
\hline \multicolumn{8}{|c|}{ Model 2} \\
\hline & $\mu_{0}$ & $\mu$ & $\sigma$ & $a$ & $\alpha$ & $\lambda_{p}$ & $\lambda_{m}$ \\
\hline Real & 0 & 0 & 0.150 & 2.500 & 1.650 & 1 & 1.500 \\
\hline $\mathrm{MCM}$ & -0.045 & 0 & 0.150 & 2.500 & 1.650 & 1 & 1.500 \\
\hline Essch & 0 & -0.727 & 0.153 & 2.500 & 1.650 & 1.750 & 0.750 \\
\hline
\end{tabular}

In Figures 5 we compare the returns real density with that obtained using Esscher transform and Mean Correcting Martingale approach.

As expected, the Mean Correcting Martingale approach involves a traslation of the original density while the Esserch Transforms involves a distorsion on tails. The behaviour of call prices for the parameters in Table 1 are reported in Figure 6 where the prices obtained by Mean Correcting Martingale approach seems to be higher than those with Esscher transforms when $\alpha$ increases.

In order to study deeply the effect of the $\alpha$ parameter on prices, we report in Figure 7 the following quantity

$$
\frac{\partial \text { Call }^{\text {theo }}(\alpha)}{\partial \alpha}=\frac{\text { Call }^{\text {theo }}(\alpha+\epsilon)-\text { Call }^{\text {theo }}(\alpha-\epsilon)}{2 \epsilon} .
$$

From Figure 7, it is possible to see that, for short maturities, under both measures, the behaviour of prices seems to be similar for varying $\alpha$ even if, as shown in Figure 

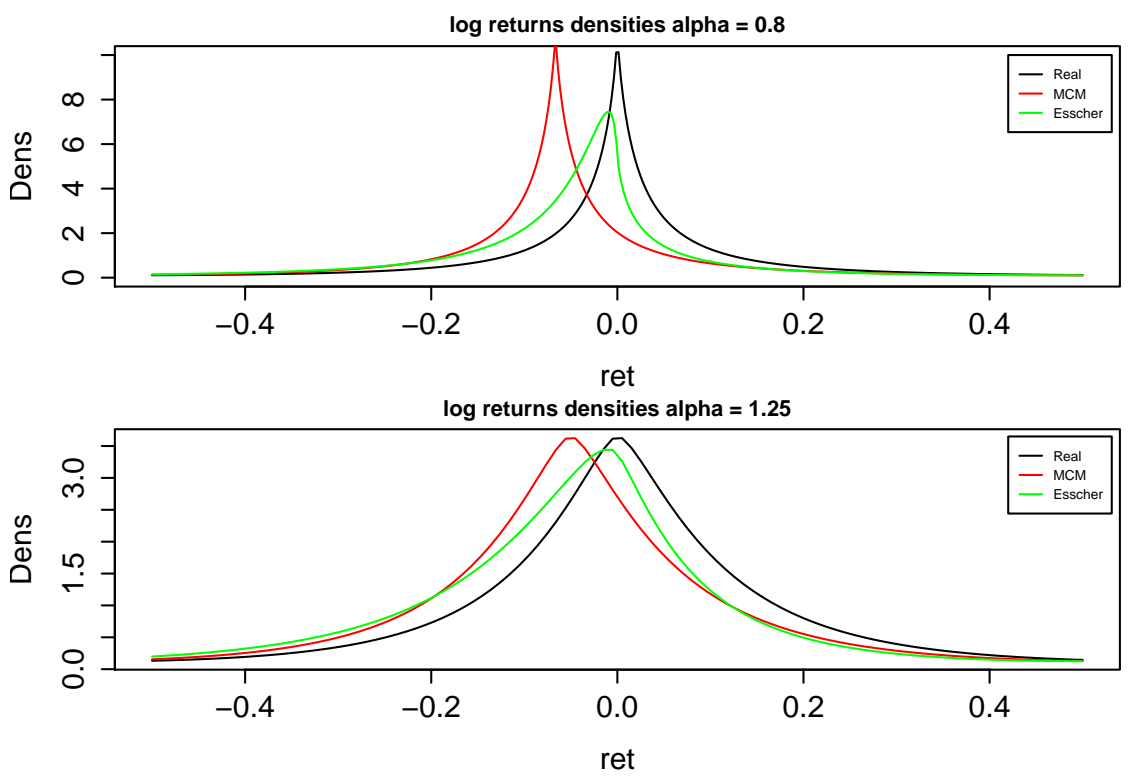

log returns densities alpha $=\mathbf{1 . 6 5 0}$

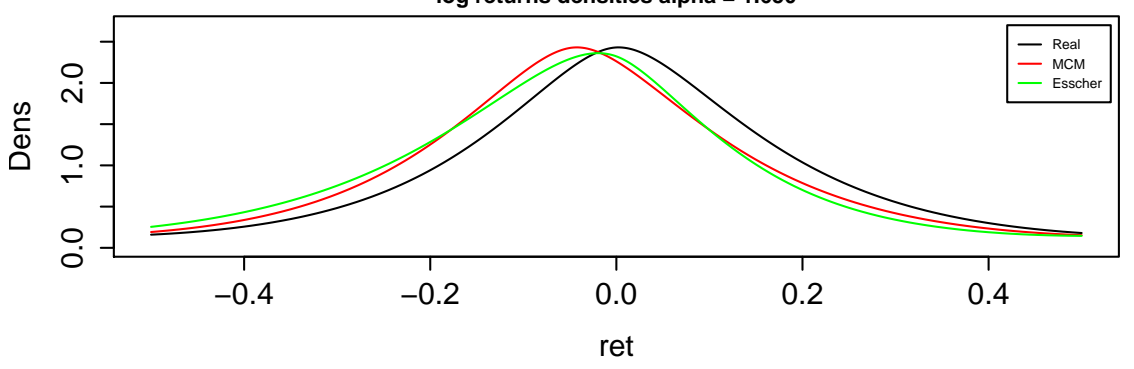

Fig. 5 Comparison between real and equivalent martingale measure.

5, the Mean Correcting Martingale measure is just a translation of the real density while the Esscher transform changes the tails of the distribution.

We conclude this Section with an analysis on real data. The dataset is composed by the mid call prices with maturity 15 days on APPLE observed at 31 december 2015 and the moneyness ranging from 0.6 to 1.4. We estimate the MixedTS parameters using the AMGNE approach introduced in Section 4 and we compare it with the Variance Gamma model. Results are reported in Table 2

The AMGNE approach gives us the possibility of building a statistical test in order to identify the appropriate model for the data. Indeed we are able to construct a likelihood ratio test where the Null hypothesis $H_{0}$ is the following:

$$
\mathcal{H}_{0}: \alpha=2 \text {. }\{\text { Market prices follow a Variance Gamma model. }\}
$$

against the Alternative hypothesis $H_{1}$ :

$\mathcal{H}_{1}: \alpha \in(0,2)$ \{Market prices follow a MixedTS model. $\}$. 

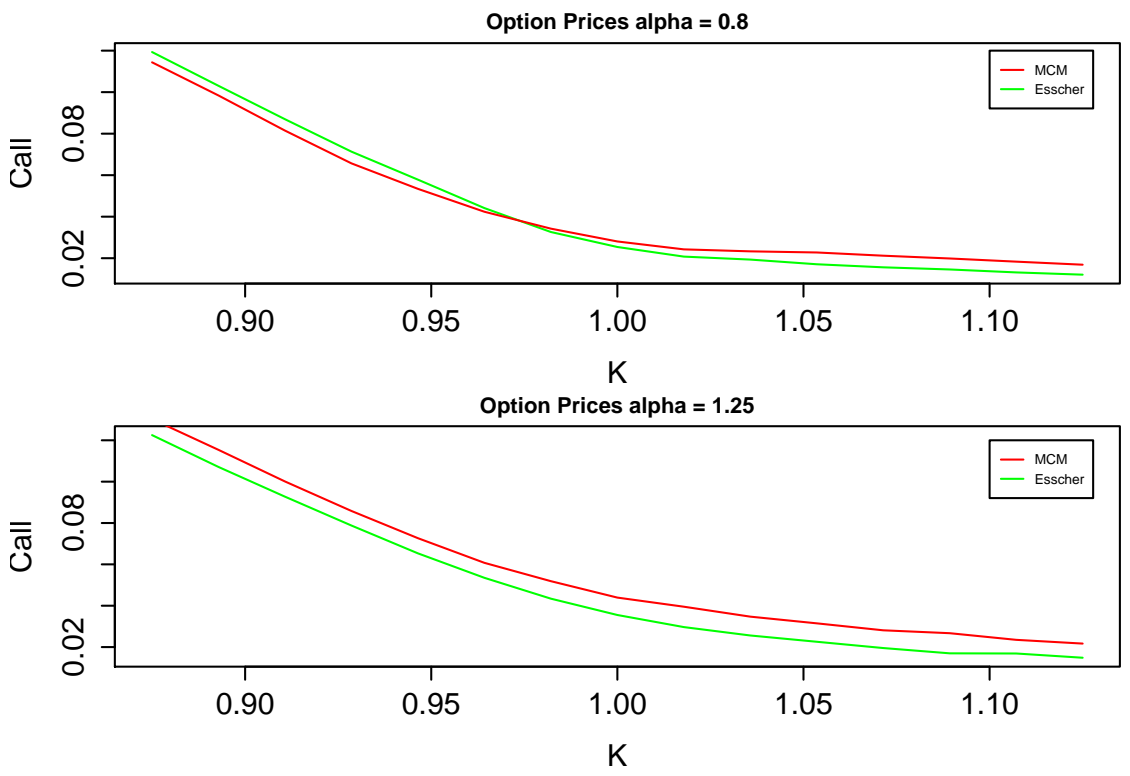

Option Prices alpha $=1.65$

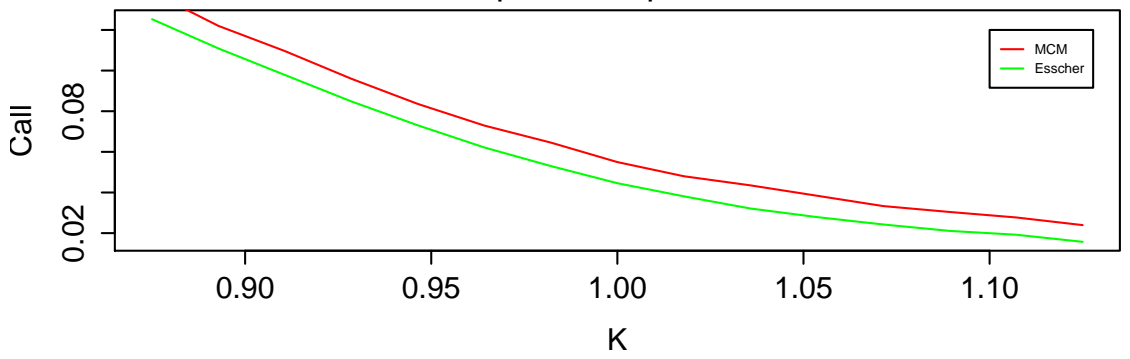

Fig. 6 Call prices behaviour for varying $\alpha$ with maturity 15 days

Table 2 Estimated parameters using real call options on APPLE with maturities 2 weeks.

\begin{tabular}{|c|c|c|c|c|c|}
\hline \multicolumn{3}{|c|}{ Exponential Mixed Tempered Stable model } & \multicolumn{3}{|c|}{ Exponential Variance Gamma model } \\
\hline param & $\operatorname{Essch}_{P}$ & $\mathrm{MCM}$ & param & $\operatorname{Essch}_{P}$ & MCM \\
\hline$\mu_{0}$ & $0.0230(0.0002)$ & 0.0207 (NA) & $\mu_{0}$ & $0.0143(0.0003)$ & $0.0120(\mathrm{NA})$ \\
\hline$\mu$ & $-0.5474(0.0005)$ & $-0.9999(\mathrm{NA})$ & $\mu$ & $0.1109(0.0015)$ & $-0.5653(\mathrm{NA})$ \\
\hline$\sigma$ & $0.0300(0.0001)$ & $0.0363(\mathrm{NA})$ & $\sigma$ & $0.0875(0.0004)$ & $0.0374(\mathrm{NA})$ \\
\hline$a$ & $2.5521(0.0067)$ & 6.5945 (NA) & $a$ & $1.2376(0.0022)$ & 4.8871 (NA) \\
\hline$\alpha$ & $1.9562(0.0057)$ & 1.8793 (NA) & - & - & - \\
\hline$\lambda_{p}$ & $2.9160(0.0119)$ & 4.6575 (NA) & - & - & - \\
\hline$\lambda_{m}$ & $1.3380(\mathrm{NA})$ & 0.0233 (NA) & - & - & - \\
\hline$\beta$ & $0.9850(0.3220)$ & 319 (NA) & $\beta$ & $0.6354(0.1502)$ & 319 (NA) \\
\hline$\gamma$ & $0.0287(0.0144)$ & 0.0766 (NA) & $\gamma$ & $0.0175(0.0121)$ & 0.0864 (NA) \\
\hline$\rho$ & $4.7 \mathrm{e}-07(0.046)$ & 0.3866 (NA) & $\rho$ & $1.1 \mathrm{e}-06(0.0560)$ & 0.3458 (NA) \\
\hline $\log \mathrm{L}$ & 212 & 172 & $\log \mathrm{L}$ & 169 & 163 \\
\hline
\end{tabular}




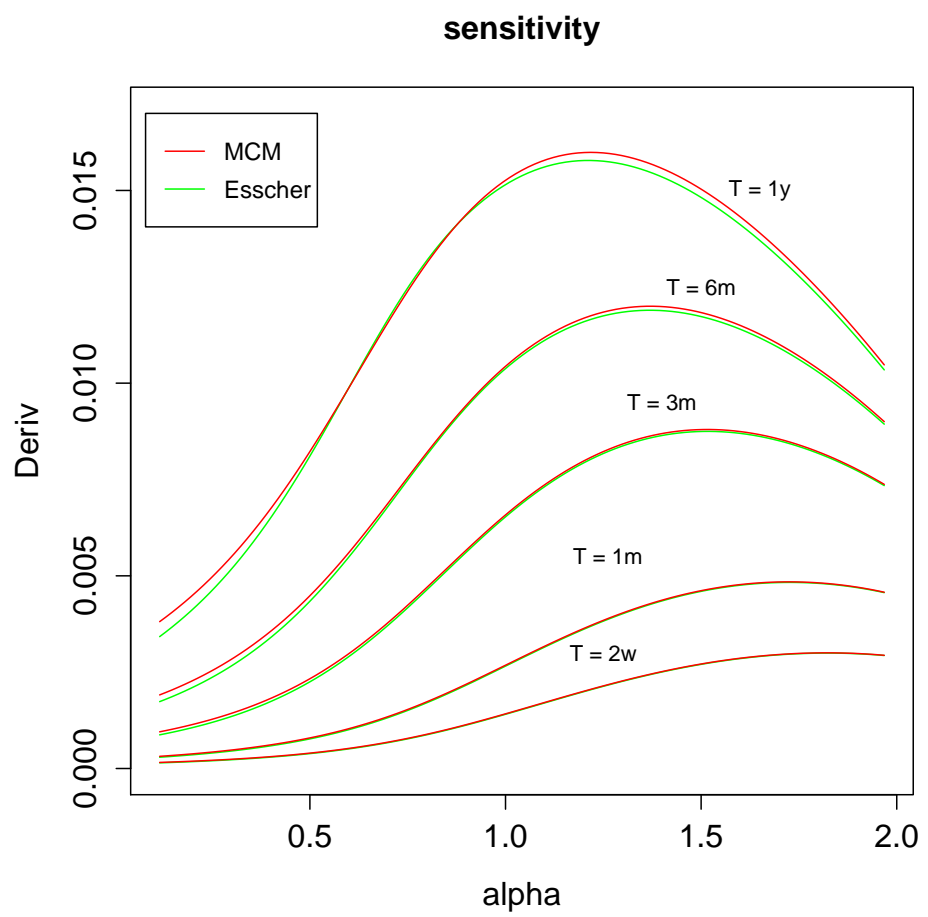

Fig. 7 Sensitivity of call prices with respect to $\alpha$ and time to maturity.

In this case, the test statistic LR is given as:

$$
\mathrm{LR}=-2\left(l_{0}-l_{1}\right) \sim \chi_{3}^{2}
$$

where $l_{0}$ and $l_{1}$ are the log-likelihood of the Variance Gamma and the MixedTS models respectively. Looking at the results in Table 2, the LR statistic is 86 for the Esscher Transform and 18 for the Mean Correcting Martingale measure. I both cases we can conclude that the Null hypothesis $H_{0}$ can be rejected in our dataset.

In the Essher transform case, we observe that the value of $\rho$ is close to zero and consequently the pricing error seems to be additive. Moreover the value of $\beta \approx 1$ suggests us that the error pricing can be a double exponential random variable while, in the Mean Correcting Martingale case, the error seems to be an uniform random variable.

\section{Conclusion}

In this paper we presented a general calibration model that encompasses well-known methodologies. We discussed option pricing for Mixed Tempered Stable distributed $\log$ returns and explain step by step how to use the new introduced $\mathrm{R}$ package that allows the user to perform calibration methodology selection through likelihood ratio tests. 


\section{References}

T. Arnold and T. F. Crack. A practical guide to gmm (with applications to option pricing). Available at SSRN 268828, 1999.

G. E. P. Box and G. C. Tiao. A further look at robustness via bayes's theorem. Biometrika, 49(3/4):pp. 419-432, 1962.

P. Carr and D. Madan. Option valuation using the fast fourier transform. Journal of computational finance, 2(4):61-73, 1999.

P. Carr and L. Wu. Time-changed lévy processes and option pricing. Journal of Financial Economics, 71(1):113 - 141, 2004a.

P. Carr and L. Wu. Time-changed lévy processes and option pricing. Journal of Financial economics, 71(1):113-141, 2004b.

P. Christoffersen and K. Jacobs. The importance of the loss function in option valuation. Journal of Financial Economics, 72(2):291-318, 2004.

E. Eberlein and D. B. Madan. Sato processes and the valuation of structured products. Quantitative Finance, 9(1):27-42, 2009.

C. S. Forbes, G. M. Martin, and J. Wright. Inference for a class of stochastic volatility models using option and spot prices: Application of a bivariate kalman filter. Econometric Reviews, 26(2-4):387-418, 2007.

H. U. Gerber, E. S. Shiu, et al. Option pricing by esscher transforms. Transactions of the Society of Actuaries, 46(99):140, 1994.

A. Hurn, K. Lindsay, and A. McClelland. Estimating the parameters of stochastic volatility models using option price data. Journal of Business 86 Economic Statistics, (just-accepted):00-00, 2014.

N. L. Johnson and S. Kotz. Distributions in Statistics. Wiley, New York, NY, 1970. In four volumes.

H. Li, M. T. Wells, and L. Y. Cindy. A bayesian analysis of return dynamics with lévy jumps. Review of Financial Studies, 21(5):2345-2378, 2008.

E. Rroji and L. Mercuri. Mixed tempered stable distribution. Quantitative Finance, 15(9):1559-1569, 2015.

K. I. Sato. Lévy processes and infinitely divisible distributions. Cambridge university press, 1999.

W. Schoutens. Lévy Processes in Finance: Pricing Financial Derivatives. John Wiley and Sons, 2003.

W. Schoutens, E. Simon and J. Tistaert A Perfect Calibration! Now What?. Wilmott Magazine, 8:66-78, 2004.

M. Subbotin. On the law of frequency of error. Mathematicheskii Sbornik, 31:296301, 1923.

P. Tankov. Financial modelling with jump processes, volume 2. CRC press, 2003.

G. C. Tiao and D. R. Lund. The use of olumv estimators in inference robustness studies of the location parameter of a class of symmetric distributions. Journal of the American Statistical Association, 65(329):pp. 370-386, 1970. 